psychopraxis.neuropraxis 2016 1 19:203

DOI 10.1007/s00739-016-0359-1

Online publiziert: 14. November 2016

(c) Springer-Verlag Wien 2016

CrossMark

Sehr geehrte Kollegin,

sehr geehrter Kollege!

In dieser Ausgabe der Zeitschrift psychopraxis.neuropraxis finden Sie Fallbeispiele aus der täglichen Praxis zu unterschiedlichen psychiatrischen und neurologischen Themen.

Die Alkoholdemenz oder die Wernicke-Enzephalopathie zum Beispiel können in Folge einer Alkoholkrankheit auftreten. Die Bedeutung der Alkoholdemenz wird allgemein meist unterschätzt, bei Patienten unter 65 Jahren ist sie eine der häufigeren Demenzerkrankungen.

Die Wernicke-Enzephalopathie hingegen ist ein sehr akutes Geschehen, welches sofortige diagnostische und therapeutische Maßnahmen erfordert. Durch eine entsprechende Therapie können die Auswirkungen gut behandelt und limitiert werden.

Das Thema therapeutische Behandlung mit Cannabis nimmt in Fachkreisen und in der Laienpresse einen immer größer werdenden Platz ein. Im Artikel „Die Auferstehung: Der Einfluss von Cannabis auf den Verlauf einer psychotischen Erkrankung“ wird die mögliche Auswirkung von Cannabisabusus ausführlich diskutiert. Besonders hervorzuheben ist, dass Jugendliche mit Cannabismissbrauch im Vergleich zu cannabisabstinenten ein deutlich höheres Risiko für die Entwicklung einer psychotischen Störung aufweisen.

Anhand eines weiteren Fallbeispiels wird das systematische diagnostische Vorgehen bei Verdacht auf multiple Sklerose beschrieben. Die neuen zusätzlichen bildgebenden Verfahren sind sowohl für die Diagnose als auch für den Verlauf und das therapeutische Monitoring von großer Bedeutung.

\title{
Josef Marksteiner
}

Abteilung für Psychiatrie und Psychotherapie A, Tirol Kliniken GmbH - Landeskrankenhaus Hall, Hall in Tirol, Österreich

\section{Aus der täglichen Praxis}

\section{Kongresse und Preisverleihungen}

Am 14.-15. November 2016 hat in Hall in Tirol die 29. Jahrestagung der Österreichischen Alzheimergesellschaft (ÖAG) mit 180 Teilnehmern aus Österreich und Deutschland stattgefunden.

In Vortragsblöcken mit verschiedenen Referenten konnte sich ein interessiertes Publikum an zwei Tagen über Themen wie „Demenz interdisziplinär“, „medikamentöse Therapiestrategien bei Demenz, Kognition und Verhalten“, „alternative Therapiestrategien“ und „Neues aus der Diagnostik" informieren.

Bereits zum 4. Mal wurde im Rahmen der ÖAG-Tagung die Young Investigator Session abgehalten.

Zehn junge Wissenschaftler präsentierten in Kurzvorträgen ihre Arbeit und stellten sich anschließend einer Diskussion. Schlussendlich konnte an fünf Kandidaten der 4. Young Investigator Award verliehen werden. Diese Plattform bietet somit jungen Wissenschaftlern die Möglichkeit, ihre Forschungsergebnisse einem Fachpublikum vorzustellen.

Im nächsten Jahr wird die 30. Jahrestagung der Österreichischen Alzheimergesellschaft im Oktober in Salzburg stattfinden.

Ich wünsche Ihnen mit dieser Ausgabe der psychopraxis.neuropraxis ein informatives Lesevergnügen und interessante Anregungen für Ihren medizinischen Alltag.

Mit besten Grüßen

Josef Marksteiner

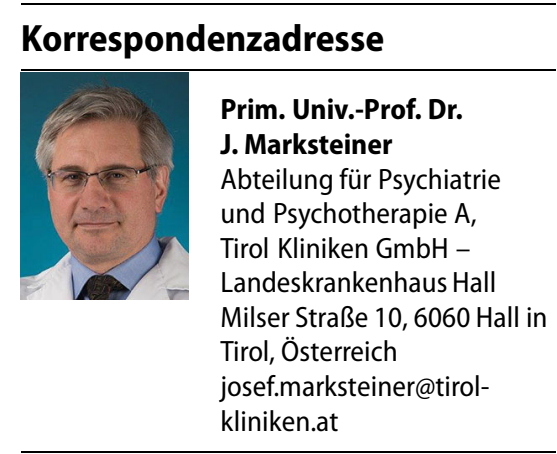

Interessenkonflikt. J. Marksteiner gibt an, dass kein Interessenkonflikt besteht. 